\title{
Algoritmo genético para calibração automática de um simulador de tráfego em rodovias de pista simples
}

\author{
C. Y. Egami ${ }^{1}$ e J. R. Setti ${ }^{2}$ \\ Universidade de São Paulo, Escola de Engenharia de São Carlos, São Carlos, São Paulo, Brasil
}

L. R. Rilett ${ }^{3}$

University of Nebraska - Lincoln, Department of Civil Engineering, Lincoln, Nebraska, EUA

Artigo Recebido: 10/09/2004 - Aprovado: 02/05/2005

RESUMO: Este trabalho relata o desenvolvimento de um sistema automático para calibração do modelo de simulação de tráfego em rodovias de pista simples TRARR, baseado em um algoritmo genético. O processo de calibração consiste em procurar valores para alguns parâmetros selecionados do simulador, de tal modo que a diferença entre as correntes de tráfego simuladas e as observadas em campo sejam minimizadas. Os parâmetros escolhidos para a calibração estão relacionados principalmente ao comportamento dos motoristas e, por isso, de difícil calibração. As diferenças entre a simulação e o fluxo real foram determinadas em termos de duas medidas de desempenho: a velocidade média dos veículos ao percorrer o trecho estudado e a proporção de veículos viajando em pelotões. O processo proposto calibra o modelo simultaneamente para cinco trechos diferentes, a fim de encontrar o conjunto de parâmetros calibrados que melhor represente uma rodovia típica brasileira. Os dados usados foram coletados em rodovias localizadas na região centro-norte do estado de São Paulo. Os resultados obtidos demonstram que o sistema proposto para a calibração funciona adequadamente e que a diferença média encontrada foi $2,75 \%$ e a diferença máxima, $4,09 \%$.

ABSTRACT: This papers reports the development of a system for automatic calibration of the TRARR simulation model, based on a genetic algorithm. The calibration process requires searching for values of selected model parameters, as to minimize the differences between simulation results and the observed data. The parameters selected for calibration are mainly related to driver behavior and thus, of difficult calibration. The differences between the simulation results and the observed data were assessed by two performance measures: average travel speed and percent vehicles traveling in platoons. The proposed procedure calibrates the model simultaneously for five different highway sections, to find the set of calibrated parameters that can best represent a two-lane highway in Brazil. Data for the calibration were collected in five different locations in the north central region of the state of São Paulo. The results indicate that the proposed calibration procedure is adequate; the mean difference between simulated and observed traffic streams was found to be $2,75 \%$ and the maximum difference, $4,09 \%$.

\section{INTRODUÇÃO}

Os modelos de simulação são uma ferramenta extremamente difundida e poderosa para a análise operacional de correntes de tráfego rodoviário. Os modelos de simulação têm desempenhado um papel importante no desenvolvimento do Highway Capacity Manual desde a versão publicada em 1985. Uma das maiores vantagens da simulação, oposta à observação real do tráfego, é a possibilidade que o usuário tem de controlar as condições em que o fluxo ocorre (McLean, 1989). No entanto, para que os resultados das simulações sejam confiáveis, é imprescindível que o modelo seja capaz de reproduzir adequadamente o comportamento da corrente de tráfego. Para isso, ele deve ser submetido a um processo de calibração através do qual seus parâmetros internos são ajustados de forma que as diferenças entre os resultados da simulação e as observações feitas no sistema real sejam minimizadas.

Em função da complexidade e do grande número de parâmetros usados nos modelos de simulação de tráfego, as abordagens tradicionais para calibração costumam ser excessivamente trabalhosas, tediosas, demoradas e pouco eficientes. Este artigo apresenta um processo automático de calibração do simulador TRARR (Hoban et al.; 1991) baseado num algoritmo genético, no qual o modelo é calibrado simultaneamente para cinco trechos diferentes de rodovia, de tal modo que o modelo calibrado seja capaz de represen- 
tar adequadamente rodovias com características similares àquelas cujos dados foram usados no processo de calibração.

A aplicabilidade de algoritmos genéticos na calibração de modelos de simulação de tráfego já foi demonstrada para os modelos TSIS (Cheu et al., 1998; Rilett e Kim, 2001) e PARAMICS (Lee et al., 2001); no entanto, os autores desconhecem uma aplicação de algoritmos genéticos na calibração de modelos de simulação de rodovias de pista simples, o que reforça o caráter inovador desta iniciativa.

\section{O MODELO TRARR}

O modelo de simulação calibrado é o TRARR, abreviação de TRAffic on Rural Roads, desenvolvido na Austrália pelo ARRB Transport Research Ltd (Hoban et al., 1991). A versão que está sendo usada neste estudo é a última produzida, a versão 4.0 de 1991 . Embora seu desenvolvimento tenha sido interrompido pelo ARRB no final da década de 1990, o TRARR foi escolhido para esta pesquisa por ser um modelo robusto, confiável e de execução rápida (Koorey, 2002; Botha et al., 1993), qualidades desejáveis para o sistema automático de calibração proposto.

$O$ TRARR utiliza quatro arquivos para entrada de dados: TRAF (arquivo de dados de tráfego), ROAD (arquivo de dados sobre a geometria de rodovia), OBS (arquivo de dados sobre os pontos onde devem ser obtidos os resultados) e VEHS (arquivo de dados sobre as características veiculares). O processo de simulação inicia-se com a leitura dos arquivos de dados sobre a geometria do rodovia e as características da corrente de tráfego simulada. O progresso dos veículos ao longo da rodovia é revisto a intervalos de um segundo, em que o programa modifica a posição no trecho, velocidade, aceleração, manobra e "estado" (livre, ultrapassando e em pelotão) de cada veículo. Os resultados são armazenados no arquivo OUT, gerado ao final de cada simulação (Hoban et al., 1991).

\section{CALIBRAÇÃO DE MODELOS DE SIMULAÇÃO}

A calibração é o processo de ajuste dos valores dos parâmetros do modelo de simulação usando dados observados em campo para que ele possa representar realistícamente componentes específicos do sistema modelado. Devido à complexidade da calibração de modelos de tráfego, o processo é essencialmente heurístico e inclui a identificação dos valores dos parâmetros que otimizam uma ou mais medidas de ajuste (Rilett e Kim, 2001). A calibração de parâmetros de um modelo de simulação pode ser considerada como um problema de otimização em que se busca o conjunto de parâmetros que maximize uma função objetivo (Kim e Rilett, 2001).

O processo de calibração proposto utiliza um algoritmo genético e não é específico para rodovias brasileiras, podendo ser aplicado em qualquer outro país, basțando que se disponham de dados relativos à geometria da rodovia, aos tipos de veículos e ao comportamento do tráfego, já que o objetivo da calibração é minimizar as diferenças entre a corrente de tráfego real e a simulada. Os algoritmos genéticos são algoritmos de busca baseados nos mecanismos da seleção natural e da genética e diferem dos procedimentos de busca e otimização tradicionais em quatro pontos: (a) trabalham com a codificação binária dos parâmetros, não com os próprios parâmetros; (b) realizam a busca a partir de um conjunto de pontos; (c) não usam derivadas; e, (d) usam regras probabilísticas e não regras determinísticas (Goldberg, 1989).

A calibração de parâmetros de modelos de simulação utilizando algoritmo genético já foi feita em Cingapura (Cheu et al., 1998), nos Estados Unidos (Rilett e Kim, 2001; Kim e Rilett, 2001 e 2004) e Canadá (Ma e Abdulhai, 2001 e 2002). Basicamente, todos seguiram um mesmo padrão: dados coletados em campo são comparados com os resultados da simulação para verificar o desempenho do algoritmo genético. O FRESIM (calibrado em Cingapura), o TRANSIMS e o CORSIM (calibrados nos EUA), são simuladores específicos para freeways; o PARAMICS (calibrado no Canadá) pode ser aplicado tanto em área urbana como rural.

\section{O PROCESSO DE CALIBRAÇÃO PROPOSTO}

O processo de calibração proposto é baseado no algoritmo desenvolvido por Rilett e Kim (2001) para a calibração do simulador TRANSIMS. O diagrama esquemático do processo usado está apresentado na Figura 1. Existem três módulos principais no programa: módulo de controle, módulo de simulação e módulo do algoritmo genético. Esses módulos trabalham em conjunto e são responsáveis por atividades distintas. No módulo de controle são manipulados os dados de entrada e saída dos demais módulos; as simulações são executadas no módulo de simulação e a aplicação dos operadores genéticos é executada no módulo do algoritmo genético.

Para iniciar o processo de calibração, uma população inicial deve ser conhecida previamente ou gerada de forma aleatória no módulo de controle. Um conjunto de valores dos parâmetros do simulador é denominado de cromossomo. Assim, uma população inicial, formada por um determinado número de cromossomos, é usada para iniciar o processo de calibração. Neste estudo, o cromossomo é formado por 7 alelos, que correspondem aos parâmetros do simulador que são usados no processo de calibração. Os parâmetros usados na calibração foram escolhidos através de análises de sensibilidade, cuja descrição 


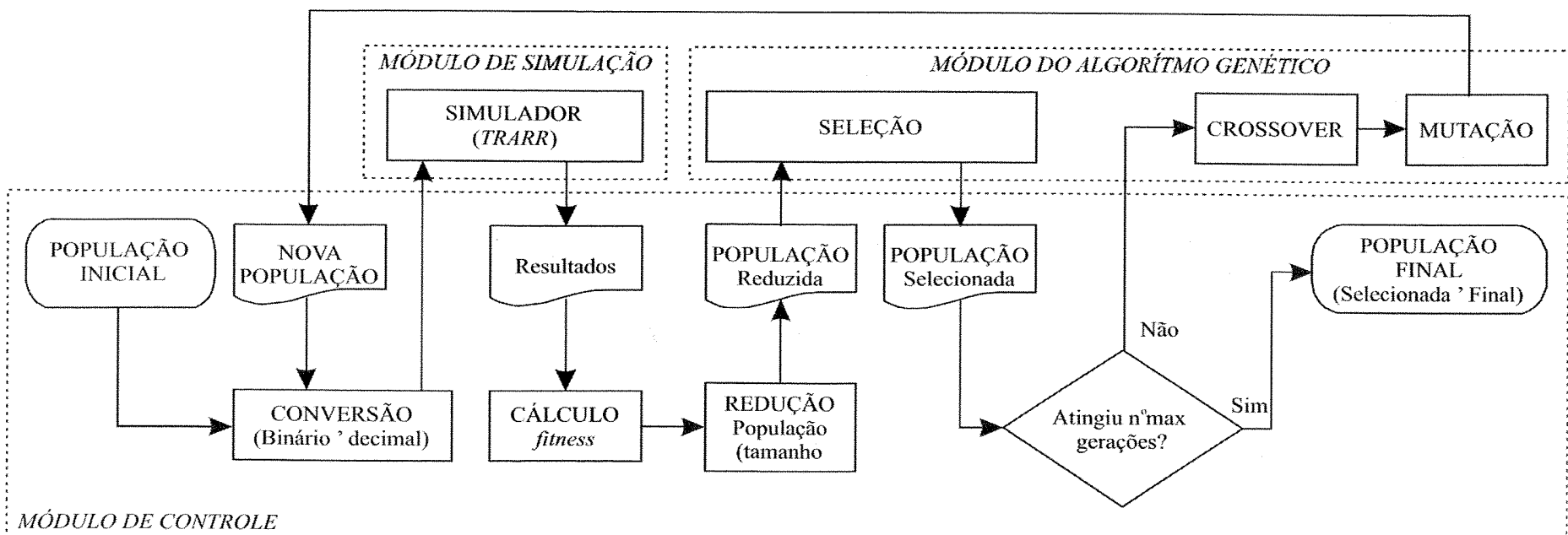

Figura 1. Diagrama esquemático do processo de calibração proposto para este estudo.

foge do escopo deste artigo. Os parâmetros selecionados foram aqueles cuja variação causa maior impacto nos resultados da simulação, em termos das medidas de desempenho escolhidas, a velocidade média e porcentagem de veículos em pelotões.

Os dois primeiros alelos do cromossomo são os parâmetros VHSFN (happy speed factor where there is no auxiliary lane) e VOSFN (overtaking speed factor when there is no auxiliary lane), que estão ligados ao comportamento dos motoristas em manobras de ultrapassagem. Os parâmetros VNP (maximum power to be used while not overtaking) $\mathrm{e}$ VXP (maximum poer to be used while overtaking) correspondem à máxima relação potência/massa que os veículos podem usar em situação normal ou durante ultrapassagens. $\mathrm{O}$ algoritmo genético busca apenas valores do VNP e do VXP para veículos pesados, já que testes realizados com o simulador indicaram que TRARR consistentemente subestima o desempenho destas categorias veiculares. Optouse, então, por usar o algoritmo genético (AG) para encontrar os valores adequados para essas duas relações potência/massa. O quinto alelo é o parâmetro LAG (agression number), que está relacionado com a agressividade do motorista - os motoristas menos agressivos só ultrapassam nos locais onde existem faixas adicionais. $O$ último alelo é o parâmetro $V M F$ (mean desired speed), que é a velocidade média desejada para cada categoria veicular simulada. Apenas o valor de VMF para caminhões é usado no AG, já que, ao contrário dos automóveis, as limitações no desempenho dos caminhões impede que se adote, como velocidade média desejada, a velocidade correspondente ao $85^{\circ}$ percentil.

O processo de calibração consiste em determinar os valores correspondentes a estes cinco parâmetros que produzem os melhores resultados da simulação.

\subsection{Algoritmo genético para calibração do TRARR}

O algoritmo genético implementado neste trabalho é composto por oito etapas que serão descritas a seguir.

\subsubsection{Etapa 1: Inicializar a população e estabelecer os parâmetros}

Cada cromossomo é representado, dentro do módulo do algoritmo genético, por uma sequiência binária. Esta seqüência é composta por conjuntos de bits representando os parâmetros a serem calibrados. Cada cromossomo $X_{i}$ tem $n$ bits, em que cada parâmetro ocupa determinado número de bits nesse cromossomo. Assim, uma população inicial de $P$ cromossomos é formada por uma string binária de tamanho $B$, determinado por:

$B \geq \log _{2}\left(\frac{x_{\max }-x_{\min }}{D}+1\right)$

em que $x_{\max }$ é o valor máximo da variável analisada, $x_{\text {min }}$ é o valor mínimo e $D$ é a precisão adotada. Por exemplo, o parâmetro $V H S F N$ de uma dada classe veicular, que pode variar entre 0 e 1 , seria representado por uma string binária de 7 bits, se a precisão $D$ for admitida como sendo 0,01, já que a Equação 1 fornece $B$ igual a 6,6582 .

No módulo de controle, essa string binária é decomposta e convertida em valores decimais que representam o conjunto de parâmetros da solução sendo analisada. Para determinar o valor decimal do parâmetro a ser usado na simulação usa-se a equação:

$x=x_{\min }+A \cdot\left(\frac{x_{\max }-x_{\min }}{2^{B}-1}\right)$

em que $A$ é o valor decimal correspondente ao vetor binário. Por exemplo, o valor decimal obtido com a Equação 2 para uma substring 1101010 que representa o VHSFN para uma dada classe veicular é 0,83 .

Cada cromossomo é formado por 328 bits, assim divididos: VOSFN (para 14 categorias veiculares), 70 bits; VHSFN (14 categorias), 98 bits; VNP e VXP (6 categorias), 36 bits cada; LAG (14 categorias), 56 bits; e VMF (4 categorias), 32 bits.

A população inicial pode ser determinada aleatóriamente pelo programa ou fornecida pelo 
usuário. Neste estudo, a população inicial foi de 300 cromossomos, cada um deles sendo uma sequência binária gerada aleatoriamente pelo programa.

\subsubsection{Etapa 2: Executar simulação}

Após a conversão dos vetores em valores decimais, o módulo de controle prepara um arquivo VEHS com os novos valores (decimais) dos parâmetros sendo recalibrados e o simulador é executado no módulo de simulação. O módulo de simulação é executado para cada cromossomo da população.

\subsubsection{Etapa 3: Avaliar os resultados}

Ao término da simulação, os resultados obtidos serão organizados e manipulados no módulo de controle para o cálculo do fitness, que reflete a qualidade dos resultados da simulação, pois mede o ajuste dos valores obtidos pelo algoritmo genético em relação aos valores observados. A função utilizada foi:

$$
\text { Fitness }=A \cdot e^{-B\left(M A E R_{i}\right)}
$$

em que: $A$ e $B$ são constantes iguais a 100 e 5 , respectivamente, e MAER (mean absolute error ratio) é a diferença média obtida em cada geração $i$, que é calculada por:

$M A E R_{i}=N^{-1} \cdot \sum_{j=1}^{N}\left(\frac{\left|V_{j}^{O}-V_{j}^{E}\right|}{V_{j}^{O}}\right)$

em que $V_{j}^{O}$ é o valor observado da variável $j$ analisada, $V_{j}^{E}$ é o valor simulado da variável $j$ analisada e $N$ é o número de variáveis analisadas. A função fitness é definida de forma a variar entre 0 e 100 , de tal forma que, quanto mais próximo de 100 , menor é a diferença média da geração sendo analisada.

\subsubsection{Etapa 4: Realizar a seleção}

O valor total da função fitness $(\mathrm{F})$ da população é determinado pelo somatório dos valores individuais do fitness de cada cromossomo. Em seguida, tanto o valor total do fitness como os valores individuais são enviados para o módulo do algoritmo genético onde servem de base para a escolha dos cromossomos que serão mantidos na próxima geração, através do operador genético seleção. A probabilidade de um cromossomo ser selecionado é proporcional à relação entre o valor do seu fitness e o valor total da função fitness. Essa relação é usada na seleção dos cromossomos que formarão as novas populações ao longo das gerações, ou seja, quanto maior a relação, maior a probabilidade do cromossomo participar da geração seguinte. A seleção é um dos três operadores genéticos aplicados no módulo do algoritmo genético; os outros dois são: crossover (troca de material genético) $\mathrm{e}$ mutação.

\subsubsection{Etapa 5: Realizar troca de material genético (crossover)}

Na nova população formada, a aplicação do operador genético crossover provoca a troca de material genético, que tem probabilidade de ocorrência fixada previamente. Para a população de $P$ cromossomos, pode-se selecionar até $P / 2$ pares de cromossomos a serem submetidos a essa troca e que formarão $C$ cromossomos descendentes (sendo $C$ d" $P / 2$ ). A parte dos cromossomos que será trocada é selecionada aleatoriamente. A probabilidade de ocorrência da troca de material genético adotada neste trabalho foi $50 \%$.

\subsubsection{Etapa 6: Realizar mutação}

A mutação, outro operador genético usado, ocorre em função de probabilidade também fixada previamente, sendo que todos os cromossomos têm chances de sofrerem mutação. Assim, se determinado cromossomo for selecionado para sofrer a mutação, um ponto em sua sequiência binária é escolhido aleatoriamente e o seu valor é trocado de 0 para 1 ou vice-versa. Assim, podem ser formados $M$ cromossomos mutantes (sendo $M$ d" $P+C$ ). Neste trabalho, adotou-se uma probabilidade de $30 \%$ para a ocorrência de uma mutação. Ao final da aplicação dos operadores genéticos, passa-se a ter uma nova geração formada por $(P+C+M)$ cromossomos.

\subsubsection{Etapa 7: Executar simulação com a nova população de descendentes}

Os cromossomos formados após a troca do material genético e a mutação são enviados para o módulo de controle, onde são transformados em valores reais para serem inseridos no arquivo de entrada do simulador. Então, já no módulo de simulação, o simulador é executado com os novos parâmetros gerados.

\subsubsection{Etapa 8: Identificar o conjunto de cromossomos sobreviventes}

Como o algoritmo trabalha com uma população de tamanho $P$ constante, é preciso eliminar o excesso de contingente populacional. Isso é feito no módulo de controle, através da ordenação desses $(P+C+M)$ cromossomos de acordo com o seu fitness. Como critério de redução da população, optou-se por eliminar cromossomos de valor intermediário de fitness, mantendo-se tanto cromossomos de maior fitness (mais aptos, que formam $80 \%$ da nova geração) quanto de menor fitness (menos aptos), já que este critério proporciona uma redução da possibilidade de convergência muito rápida do programa e evita a obtenção de um ótimo local da função fitness.

A nova geração formada por $P$ cromossomos sobreviventes é então submetida ao mesmo processo, que é repetido até que o critério de parada seja satisfeito. 


\subsubsection{Etapa 9: Verificar as regras de parada do algoritmo}

São dois os critérios de parada usados: o número máximo de gerações ou a diferença mínima entre os valores observados e simulados. Os valores para os dois critérios são estipulados pelo usuário; no caso, foram usadas 100 gerações ou diferença menor que $2 \%$. Como o modelo foi calibrado usando-se simultaneamente dados coletados em 5 trechos diferentes, a diferença considerada é a média $\bar{D}$ das diferenças encontradas para cada trecho:

$\bar{D}=\frac{\sum_{i=1}^{r} d_{i f}}{r}$

em que r é o número de trechos e dif é a diferença entre ofluxo simulado e observado, calculada por:

$d i f_{i}=\frac{\sum_{k=1}^{n} \frac{\left|o b s_{k}-\operatorname{simul}_{k}\right|}{o b s_{k}}}{n}$

em que $o b s_{k}$ e simul são, respectivamente, os valores observado e simulado da medida de desempenho $k$; e $n$ é o número de medidas de desempenho utilizadas.

\section{DADOS USADOS NA CALIBRAÇÃO}

Os dados usados na calibração do modelo foram coletados em cinco trechos de rodovias, localizados no estado de São Paulo e incluem tanto trechos concessionados como sob jurisdição do DER-SP. Os trechos estudados são: SP215, do km 133 ao km 138 (trecho 1); SP225, km 113-121 (trecho 2) e km 133141 (trecho 3); SP253, km 151-155 (trecho 4); e SP $322, \mathrm{~km}$ 382-389 (trecho 5). O sentido 1 sempre corresponde ao sentido dos marcos quilométricos; o sentido
2 é o oposto. As extensões dos trechos variam entre $4 \mathrm{~km}$ e $8 \mathrm{~km}$. Os trechos escolhidos são quase todos em tangente e os perfis verticais variam entre praticamente planos (sem restrições de ultrapassagens e declividade média de $0,5 \%$ ) a ondulados ( $42 \%$ de restrições de ultrapassagens e rampas entre 3 e $5 \%$ ). Isso pode ser visto nas Figura $2 \mathrm{a} \mathrm{e} 2 \mathrm{~b}$, que apresentam os perfis dos trechos 1 e 4.

Os dados necessários para a elaboração do arquivo com as características de cada trecho simulado (ROAD) foram obtidos dos projetos geométricos e incluem rampas (declividade e comprimento) e distâncias de visibilidade de ultrapassagem em cada sentido, determinados em intervalos de 100 metros.

Os dados necessários para a caracterização das correntes de tráfego em cada trecho (arquivo TRAF) foram coletados por meio de câmeras de vídeo, através de uma adaptação do método das placas (Robertson et al., 1994), geralmente empregado em pesquisas $\mathrm{O} / \mathrm{D}$ e amplamente usado em estudos similares ao realizado (Romana, 2004; Harwood et al., 1999; Archilla e Morrall, 1999). Quatro câmeras de vídeo digital são posicionadas na rodovia, duas na entrada e duas na saída do trecho, cada uma monitorando um sentido de tráfego. As placas dos veículos que passam são lidas em voz alta pelo observador, para que sejam gravadas na filmagem. As placas são também anotadas numa planilha. Os relógios internos das câmeras digitais são sincronizados antes da coleta de dados, o que permite saber, com precisão adequada, o instante exato de passagem de cada veículo, na entrada e na saída do trecho.

Da gravação, obtém-se posteriormente, a classificação inicial dos veículos, as velocidades desses veículos e a porcentagem de veículos em pelotões, na entrada e na saída do trecho. Para a determinação da velocidade média, usase o tempo gasto para percorrer o trecho $\Delta t_{i}(\mathrm{em} \mathrm{s})$; como a distância percorrida $\Delta L$ (em $\mathrm{km}$ ) é conhecida, a velocidade $V_{i}(\mathrm{em} \mathrm{km} / \mathrm{h})$ é a velocidade média no espaço:

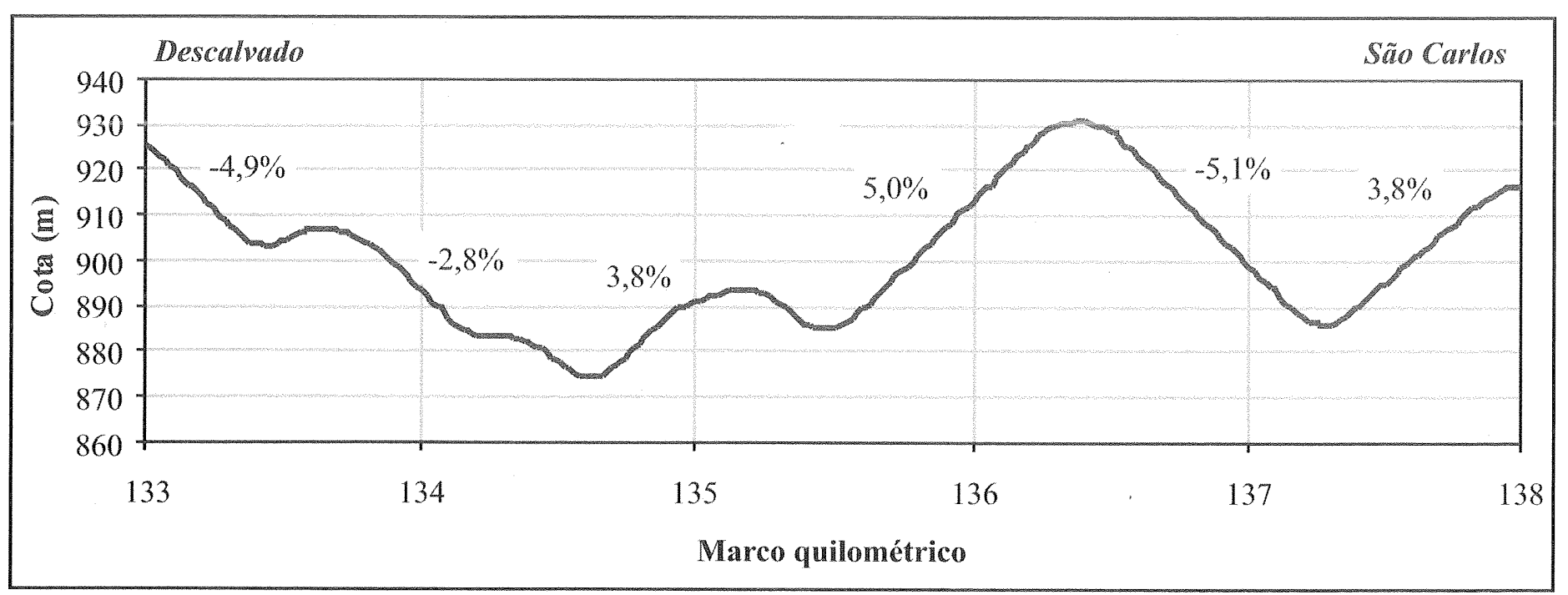

Figura 2a: Perfil vertical do trecho simulado da SP215. 


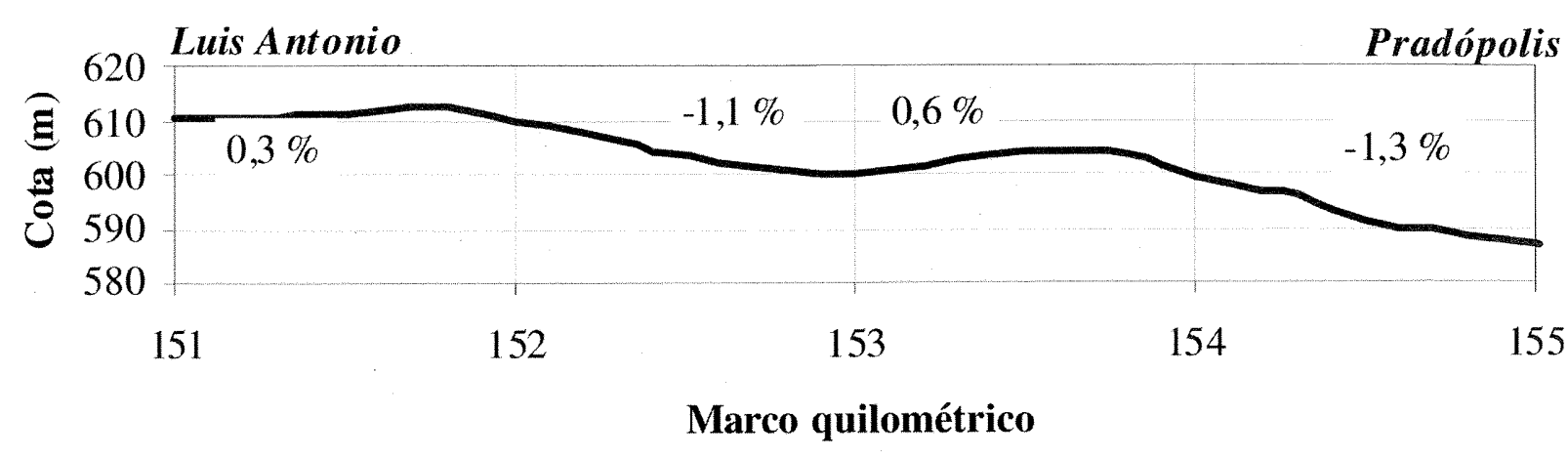

Figura 2b: Perfil vertical do trecho simulado da SP253.

$V_{i}=\frac{\Delta L}{\Delta t_{i}} \cdot 3600$

A velocidade média de cada classe veicular adotada (automóveis, caminhões e ônibus), usada na simulação, é a média da distribuição das velocidades assim calculadas para cada tipo de veículo.

A determinação da porcentagem de veículos em pelotões é feita a partir dos headways; se o headway em relação ao veículo anterior for menor ou igual a três segundos, considera-se que os dois viajam em pelotão.

O TRARR pode simular até 18 classes diferentes de veículos, cujos parâmetros e comportamento são diferentes. Uma análise do tráfego nas rodovias estudadas indicou que 14 classes são suficientes para representar a frota típica que trafega nas rodovias do estado de São Paulo. A Tabela 1 apresenta as 14 classes de veículos usadas nas simulações e suas características.

Tabela 1. Classes de veículos simuladas.

\begin{tabular}{lcc}
\hline Classe veicular & Massa $(\mathrm{t})$ & $\begin{array}{c}\text { Potência/massa } \\
(\mathrm{kW} / \mathrm{t})\end{array}$ \\
\hline VCL leve & 35,8 & 16,3 \\
VCL pesado & 59,0 & 10,3 \\
Rígido pesado & 21,9 & 3,6 \\
Articulado pesado & 42,2 & 3,0 \\
Articulado leve & 15,5 & 3,6 \\
Onibus & 12,0 & 10,0 \\
Rígido leve & 10,5 & 24,3 \\
Caminhonete & 2,5 & 30,0 \\
Van & 0,8 & 35,0 \\
Carro baixa potência & 1,1 & 40,0 \\
Carro média potência & 1,0 & 50,0 \\
Pick-up pequena & 1,7 & 55,0 \\
Carro médio & 1,4 & 50,0 \\
Carro esportivo & 1,1 & 90,0 \\
\hline
\end{tabular}

Um caminhão articulado é composto por uma unidade tratora e uma de transporte interligados por uma articulação; um caminhão rígido é composto por uma única unidade; e um VCL possui duas ou mais articulações, como o rodotrem, usado no transporte de cana de açúcar, e o bitrem, usado no transporte de combustíveis.

Para a simulação, as 14 classes da Tabela 1 são agregadas em três categorias: automóveis, caminhões leves e caminhões pesados. A categoria "automóveis" engloba todas as classes de carros de passeio e de utilitários; "caminhões leves" inclui os caminhões rígidos, articulados ou VCL que trafegam acima da velocidade média da corrente de tráfego; $\mathrm{e}$ "caminhões pesados" abrange aqueles que trafegam em velocidade abaixo da média, qualquer que seja a sua classe (rígidos, articulados e VCLs). A Tabela 2 mostra a composição do tráfego nos trechos estudados, de acordo com essa agregação.

Tabela 2. Caracterização do fluxo de tráfego nos trechos estudados (veic/hora).

\begin{tabular}{cccccc}
\hline \multirow{2}{*}{ Trecho } & \multirow{2}{*}{ Sentido } & \multirow{2}{*}{ Autos } & \multicolumn{2}{c}{ Caminhões } & \multirow{2}{*}{\begin{tabular}{c} 
Fluxo total \\
\cline { 4 - 5 }
\end{tabular}} \\
\cline { 4 - 5 } & & Leves & Pesados & (2 sentidos) \\
\hline 1 & 1 & 67 & 4 & 27 & 213 \\
& 2 & 77 & 8 & 30 & \\
\hline 2 & 1 & 80 & 13 & 52 & 250 \\
& 2 & 70 & 5 & 30 & \\
\hline 3 & 1 & 65 & 8 & 23 & 190 \\
& 2 & 72 & 4 & 18 & \\
\hline 4 & 1 & 39 & 20 & 55 & 235 \\
& 2 & 45 & 19 & 58 & \\
\hline 5 & 1 & 124 & 7 & 37 & 351 \\
& 2 & 138 & 9 & 36 & \\
\hline
\end{tabular}

Tabela 3. Médias das distribuições observadas da velocidade nos trechos estudados $(\mathrm{km} / \mathrm{h})$.

\begin{tabular}{ccrccc}
\hline \multirow{2}{*}{ Trecho } & \multirow{2}{*}{ Sentido } & \multirow{2}{*}{ Autos } & \multicolumn{2}{c}{ Caminhões } & \multirow{2}{*}{\begin{tabular}{c} 
Velocidade \\
\cline { 4 - 5 }
\end{tabular}} \\
\cline { 4 - 5 } & 1 & & Leves & Pesados & média \\
\hline \multirow{2}{*}{1} & 2 & 87,2 & 83,9 & 98,4 & 80,2 \\
& 1 & 90,2 & 86,7 & 70,1 & 84,3 \\
\hline \multirow{2}{*}{2} & 1 & 87,8 & 83,9 & 98,4 & 80,2 \\
& 2 & 90,2 & 86,7 & 70,1 & 84,3 \\
\hline \multirow{2}{*}{3} & 1 & 91,7 & 92,6 & 80,6 & 89,1 \\
& 2 & 86,4 & 81,0 & 60,7 & 81,2 \\
\hline \multirow{2}{*}{4} & 1 & 100,0 & 94,8 & 84,0 & 91,3 \\
& 2 & 90,2 & 85,6 & 72,8 & 81,2 \\
\hline \multirow{2}{*}{5} & 1 & 95,1 & 90,9 & 73,4 & 90,1 \\
& 2 & 86,8 & 83,1 & 74,1 & 84,1 \\
\hline
\end{tabular}


Tabela 4. Porcentagem observada de veículos em pelotões (\%).

\begin{tabular}{cccc}
\hline Trecho & Sentido & Entrada & Saída \\
\hline 1 & 1 & 19,4 & 23,0 \\
& 2 & 25,1 & 19,6 \\
\hline 2 & 1 & 30,8 & 34,8 \\
& 2 & 23,5 & 29,7 \\
\hline 3 & 1 & 22,3 & 21,8 \\
& 2 & 24,1 & 27,1 \\
\hline \multirow{2}{*}{4} & 1 & 25,6 & 20,0 \\
& 2 & 21,7 & 20,8 \\
\hline 5 & 1 & 31,6 & 33,1 \\
& 2 & 32,6 & 32,0 \\
\hline
\end{tabular}

Para cada uma das 14 classes de veículos, foi necessário atualizar os parâmetros do TRARR relativos à relação potência/massa, à massa e ao comprimento. Para as classes compreendendo automóveis, os dados foram obtidos de publicações especializadas e catálogos de fabricantes. Os modelos nacionais foram classificados dentro das categorias já existentes no TRARR por meio de uma análise de cluster, cujos detalhamento foge do escopo deste artigo.

As massas para as classes correspondentes aos caminhões foram obtidas de um estudo prévio, que analisava a distribuição das massas de caminhões em uma balança da Via Anhanguera (Demarchi, 2000), com exceção dos VCLs, uma vez que esses veículos não podiam trafegar em rodovias de pista dupla em 2000, quando o estudo anterior foi realizado. Os dados para VCLs foram coletados em uma balança na Via Washington Luiz (SP310). As relações potência/massa dos caminhões mostradas na Tabela 1 foram obtidas através do AG, conforme discutido no item 4, uma vez que testes realizados pelos autores indicaram que o TRARR tem uma tendência a subestimar a velocidade e o desempenho de caminhões pesados, mesmo quando eles trafegam isoladamente.

A Tabela 3 mostra a média das distribuições de velocidades observadas em cada trecho, por direção e por categoria veicular. A Tabela 4 mostra a porcentagem observada de veículos trafegando em pelotões, em cada trecho, por direção, na entrada e na saída de cada trecho.

\section{RESULTADOS OBTIDOS}

O programa para a calibração do simulador TRARR foi implementado na linguagem Perl (Wall et al., 2000). A população inicial foi composta por 300 cromossomos e foram usadas 100 gerações para calibrar o simulador. As medidas de desempenho escolhidas para comparar os dados observados em campo com os resultados da simulação foram a velocidade média de cada categoria veicular e a porcentagem de veículos em pelotões em cada direção de tráfego. Essas variáveis foram escolhidas por serem comparáveis às medidas de desempenho usadas no HCM2000 para rodovias de pista simples. A velocidade usadaé a velocidade média no espaço, já que é calculada em função do tempo gasto para percorrer um segmento relativamente longo de via.

No processo proposto, o AG deve buscar valores para os parâmetros que minimizem a diferença entre a corrente real e a simulada para cinco trechos diferentes. Para isso, foram 294.665 simulações dos cinco trechos diferentes de rodovia. O tempo total gasto para processamento do AG foi de 115 horas, num micro equipado com uma CPU AMD Athlon XP 2800.

A qualidade da calibração obtida com o processo proposto pode ser verificada comparando-se os fluxos observados com os simulados. Observando-se a Tabela 5 , pode-se notar que, em média, a diferença média entre a porcentagem de veículos em pelotões nas duas correntes é inferior a $1 \%$, excedendo este valor em apenas dois dos dez casos.

Tabela 5: Resultados da calibração: porcentagem de veículos em pelotões (\%).

\begin{tabular}{ccccc}
\hline Trecho & Sentido & Observado & Simulado & Diferença \\
\hline \multirow{2}{*}{1} & 1 & 19,4 & 19,3 & $-0,1$ \\
& 2 & 25,1 & 25,6 & 0,5 \\
\hline \multirow{2}{*}{2} & 1 & 30,8 & 28,3 & $-2,5$ \\
& 2 & 23,5 & 24,2 & 0,7 \\
\hline 3 & 1 & 22,3 & 22,3 & 0,0 \\
& 2 & 24,2 & 24,2 & 0,0 \\
\hline \multirow{2}{*}{4} & 1 & 25,6 & 24,7 & $-0,9$ \\
& 2 & 21,7 & 22,1 & 0,4 \\
\hline 5 & 1 & 31,6 & 32,1 & 0,5 \\
& 2 & 32,6 & 29,4 & $-3,2$ \\
\hline
\end{tabular}

Tabela 6: Resultados da calibração: velocidade média observada e simulada $(\mathrm{km} / \mathrm{h})$.

\begin{tabular}{|c|c|c|c|c|c|}
\hline \multirow{2}{*}{ Trecho } & \multirow{2}{*}{ Sentido } & & \multirow{2}{*}{ Autos } & \multicolumn{2}{|c|}{ Caminhões } \\
\hline & & & & Leves & Pesados \\
\hline \multirow[t]{4}{*}{1} & 1 & obs & 99,7 & 92,6 & 73,2 \\
\hline & & simul & 100,0 & 92,6 & 73,9 \\
\hline & 2 & obs & 85,9 & 82,9 & 67,2 \\
\hline & & simul & 82,8 & 86,7 & 65,9 \\
\hline \multirow[t]{4}{*}{2} & 1 & obs & 87,2 & 83,9 & 68,4 \\
\hline & & simul & 82,0 & 75,5 & 68,8 \\
\hline & 2 & obs & 90,2 & 86,7 & 70,1 \\
\hline & & simul & 90,2 & 87,8 & 71,1 \\
\hline \multirow[t]{4}{*}{3} & 1 & obs & 91,7 & 92,6 & 80,6 \\
\hline & & simul & 94,9 & 91,4 & 89,0 \\
\hline & 2 & obs & 86,4 & 81,0 & 60,7 \\
\hline & & simul & 84,5 & 81,0 & 51,4 \\
\hline \multirow[t]{4}{*}{4} & 1 & obs & 100,0 & 94,8 & 84,0 \\
\hline & & simul & 101,2 & 94,9 & 94,2 \\
\hline & 2 & obs & 90,2 & 85,6 & 72,8 \\
\hline & & simul & 90,4 & 85,8 & 76,2 \\
\hline \multirow[t]{4}{*}{5} & 1 & obs & 95,1 & 90,9 & 73,4 \\
\hline & & simul & 94,6 & 87,8 & 75,7 \\
\hline & 2 & obs & 86,8 & 83,1 & 74,1 \\
\hline & & simul & 86,6 & 87,0 & 79,5 \\
\hline
\end{tabular}


A Tabela 6 permite comparar a velocidade média no espaço observada e simulada, em termos das três classes veiculares usadas. A média das diferenças absolutas entre a velocidade observada e simulada para as 30 observações da Tabela 6 é de $2,7 \mathrm{~km} / \mathrm{h}$ e a diferença é superior a $5 \mathrm{~km} / \mathrm{h}$ em apenas 6 casos e, em 17 casos, é inferior a $2 \mathrm{~km} / \mathrm{h}$.

Os gráficos da Figura 3 comparam as distribuições observadas e simuladas da velocidade para cada um dos cinco trechos usados na calibração. Pode-se notar que as curvas acumuladas das distribuições obtidas são muito semelhantes. Para compará-las, foi usado o teste de Kolmogorov-Smirnov. Neste teste, as distribuições de frequiência acumuladas nos intervalos de classe de cada amostra são comparadas, através das diferenças entre as frequiências observada e simulada em cada intervalo da distribuição. A maior diferença $\left(D_{\text {máx }}\right)$ é comparada com o valor crítico $\left(D_{\text {crítico }}\right)$. Este valor é obtido para o nível de signficância ( $\alpha$ ) adotado. Como o tamanho das amostras testadas é diferente, o valor de $D_{\text {crifico }}$ é obtido por (Rohatgi, 1976):

$D_{\text {critico }}=\beta \cdot \sqrt{\frac{n_{1}+n_{2}}{n_{1} \cdot n_{2}}}$

em que $n_{1}$ e $n_{2}$ é o tamanho de cada amostra analisada e â é a variável que depende do nível de significância adotado. No caso, adotou-se um nível de significância de $5 \%$.

Os resultados dos testes mostraram que, para $\alpha=5 \%$, a distribuição de velocidades simulada não é diferente da distribuição observada, com exceção de um dos sentidos dos trechos 2,3 e 4 . Conforme pode ser visto nos gráficos da Figura 3, as diferenças entre as distribuições são muito pequenas, mas suficientes para rejeitar a hipótese nula.

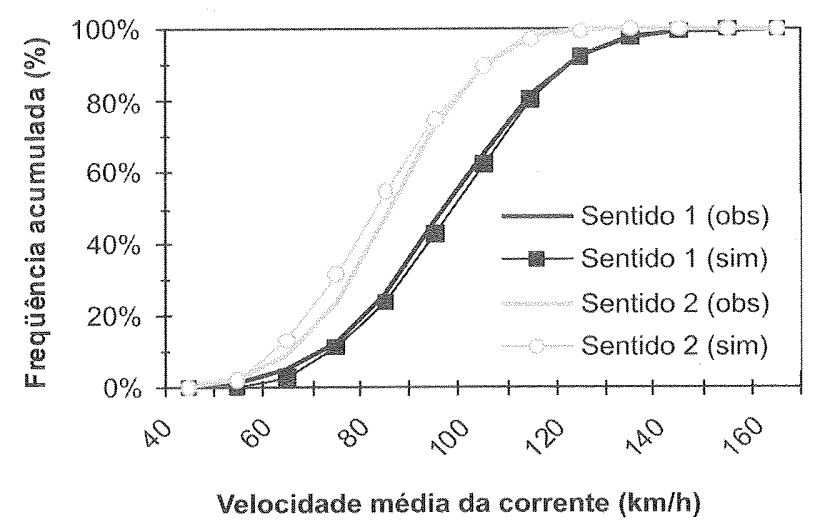

Figura 3a. Distribuições acumuladas de velocidade, trecho 1.

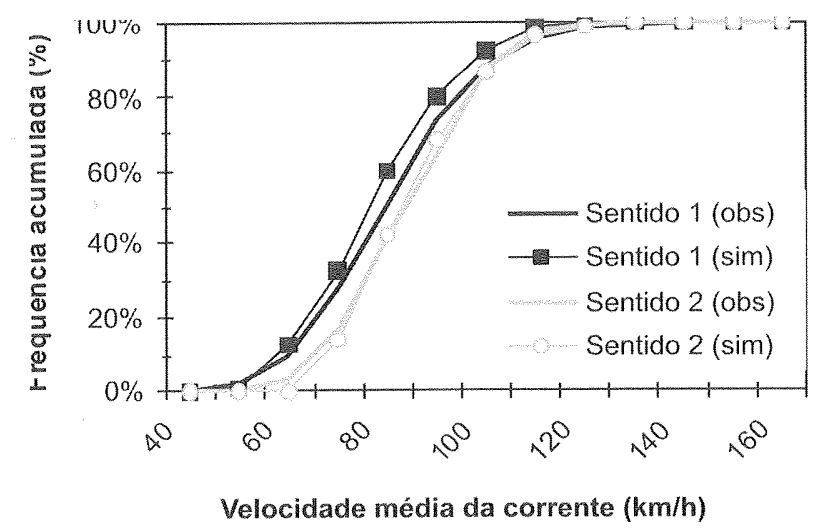

Figura 3b. Distribuições acumuladas de velocidade, trecho 2 .

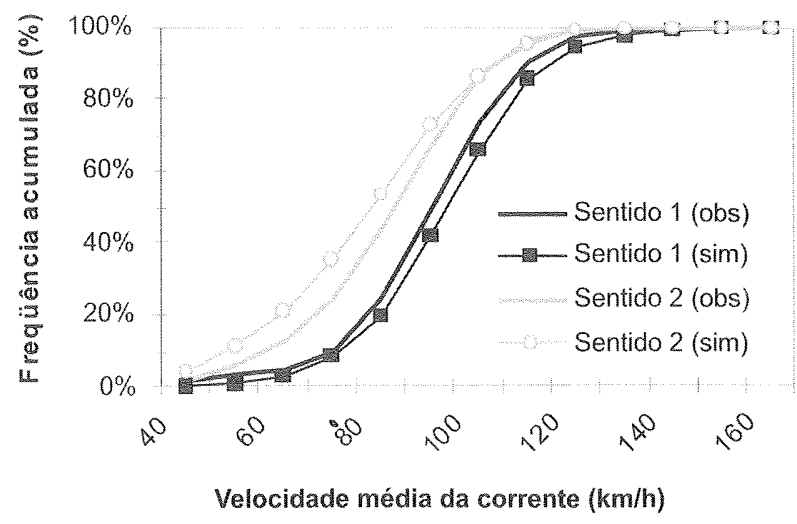

Figura 3c. Distribuições acumuladas de velocidade, trecho 3.

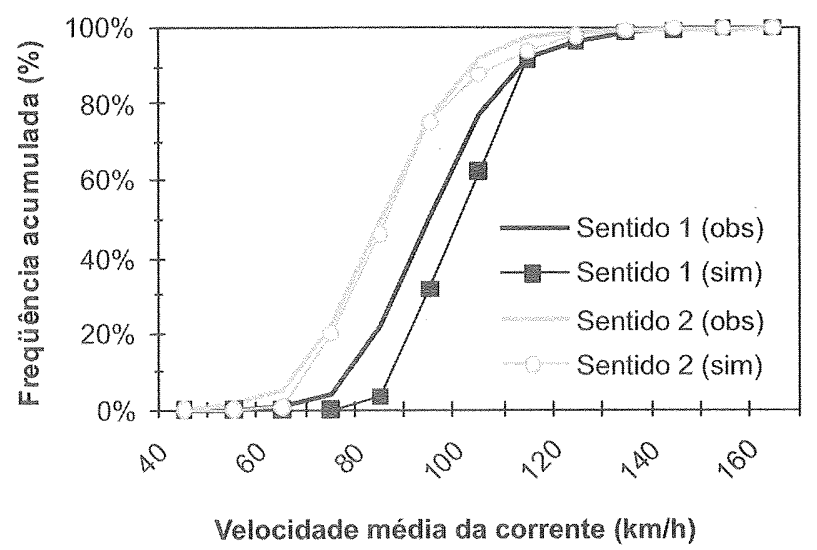

Figura 3d. Distribuições acumuladas de velocidade, trecho 4.

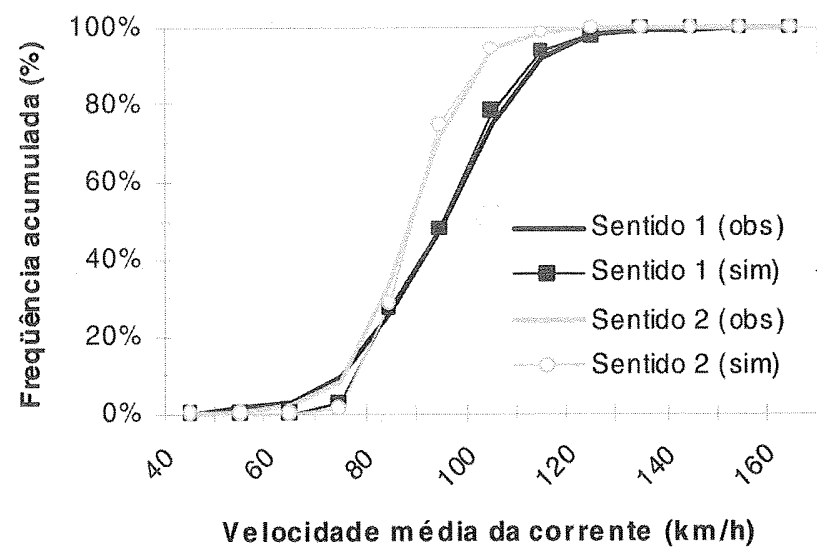

Figura 3e. Distribuições acumuladas de velocidade, trecho 5.

Tabela 7. Diferenças entre correntes simuladas e observadas.

\begin{tabular}{ccc} 
Trecho & Diferença & Fitness \\
\hline 1 & $1,50 \%$ & 92,78 \\
2 & $2,81 \%$ & 86,91 \\
3 & $4,09 \%$ & 81,50 \\
4 & $2,48 \%$ & 88,32 \\
5 & $2,87 \%$ & 86,62 \\
\hline
\end{tabular}

Existem dois fatores a que podem ser atribuídos essas diferenças. O primeiro está relacionado com a estrutura geral do AG, já que a comparação entre a corrente simulada e a observada é feita apenas em termos da média das velocidades, já que seria muito complexo (se não impossível) fazer com que o $A G$ comparasse as distribuições. O outro aspecto também está ligado ao $\mathrm{AG}$, 'uma vez que admitiu-se, na codificação do $\mathrm{AG}$, que o mesmo valor de $\mathrm{VMF}$ (velocidade desejada) deveria ser aplicado a todos os trechos. Esse aspecto deverá corrigido numa versão 
futura do $\mathrm{AG}$, que passará a fornecer valores específicos por trecho e por sentido da velocidade desejada.

O erro médio encontrado, considerando-se todos os cinco trechos estudados simultaneamente, foi de apenas $2,75 \%$, o que corresponde a fitness $=87,15$. A Tabela 7 mostra as diferenças encontradas entre correntes simuladas e observadas para cada trecho; um valor de fitness $=100$ significa que não há diferença "entre as duas correntes de tráfego.

Apesar disso, pode-se considerar que os resultados obtidos foram muito bons e muito superiores ao que se poderia esperar de uma calibração tradicional, como a realizada por Egami e Setti (2001).

\section{CONCLUSÕES E CONSIDERAÇÕES FINAIS}

Conforme comprovam os resultados apresentados neste trabalho, a abordagem proposta, que consiste em usar um algoritmo genético para calibrar um modelo de simulação usando-se, simultaneamente, diversos trechos de rodovia, mostrou-se não apenas viável, mas também capaz de encontrar valores ótimos para parâmetros cuja medição direta seria muito complexa, se não impossível. O modelo calibrado foi considerado capaz de reproduzir adequadamente o comportamento da corrente de tráfego em cinco trechos diferentes de rodovias de pista simples, considerando-se como medidas de desempenho a porcentagem de veículos em pelotão e a velocidade média ao longo do trecho.

As diferenças encontradas entre os valores simulados e os observados foram muito menores que as encontradas em calibrações realizadas por outros autores para modelos semelhantes (por ex., Harwood et al., 1999). As diferenças encontradas são pequenas, principalmente com relação à quantidade de veículos em pelotões, variável que está diretamente relacionada ao comportamento dos motoristas e que é representada no modelo por parâmetros cujos valores são impossíveis de serem medidos diretamente. As diferenças encontradas nas distribuições de velocidade foram maiores, mas não são suficientemente grandes para invalidar a calibração.

As próximas etapas da pesquisa envolvem uma alteração no $A G$ e a validação do modelo calibrado. A alteração visa obter valores específicos do parâmetro VMF (velocidade desejada) para cada trecho e direção, uma vez que a abordagem adotada (procura de um único valor de VMF que seja aplicado a todos os trechos) mostrou-se inadequada. Espera-se que esta alteração faça com que os resultados da calibração fiquem ainda melhores.

Para a validação do modelo calibrado, deverão ser usados dados obtidos em novas coletas nos trechos estudados, para que seja possível verificar quão bem o modelo calibrado é capaz de representar as correntes de tráfego. Pretende-se usar o modelo calibrado e validado na adaptação do método de análise da capacidade e nível de serviço em rodovias de pista simples do HCM2000 às rodovias brasileiras deste tipo.

\section{AGRADECIMENTOS}

O apoio financeiro para o desenvolvimento da pesquisa relatada neste artigo foi obtido junto à FAPESP (proc. 01/0279-8), sob a forma de uma bolsa de doutorado; e junto ao $\mathrm{CNPq}$, sob a forma de uma bolsa de produtividade em pesquisa (proc. 303857/2002-1), a quem os autores agradecem.

\section{REFERÊNCIAS BIBLIOGRÁFICAS}

Archilla, R. e J. Morral (1996) Traffic Characteristics on Two-Lane Highway Downgrades. Transportation Research-A, v.30, n.2, p.119-133.

Botha, J. A.; X. Zeng e E. Sullivan (1993) Comparison of performance of TWOPAS and TRARR models when simulating traffic on two-lane highways with low design speeds. Transportation Research Record, n. 1308; p. 716.

Cheu, R.; X. Jin; K. Ng, Y. Ng e D. Srinivasan (1998) Calibration of FRESIM for Singapure Expressway using genetic algorithm. ASCE Journal of Transportation Engineering, v. 124, n. 6 .

Demarchi, S. H. (2000) Influência dos Veículos Pesados na Capacidade e Nível de Serviço de Rodovias de Pista Dupla. São Carlos. 166 p. Tese (Doutorado) - Escola de Engenharia de São Carlos, Universidade de São Paulo.

Egami, C. Y. e J. R. A. Setti (2001) Recalibração do modelo TRARR para simulação de rodovias de pista simples no Brasil. In.: Transporte em Transformação V, Makron Books Ltda, Pearson Education do Brasil, Rio de Janeiro, p. 31-46.

Goldberg, D. E. (1989) Genetic Algorithms in Search, Optimization, and Machine Learning. New York, AddisonWesley Publishing Company, Inc.

Harwood, D. W.; A. D. May; I. B. Anderson; L. Leiman; A. R. Archilla (1999) Capacity and Quality of Service of TwoLane Highways - Final Report, Transportation Research Board, Washington, D.C., EUA, 166 p.

Hoban, C. J.; R. J. Shepherd; G. J. Fawcett; G. K. Robinson (1991) A Model for Simulating Traffic on Two-Lane Rural Roads - User Guide and Manual for TRARR Version 3.2. Victoria, Australia, ARRB.

Kim, K. e L. R. Rilett (2004) A Genetic Algorithm Based Approach to Traffic Micro-simulation Calibration Using ITS Data. In: Transportation Research Board $83^{\text {rd }}$ Annual Meeting, Washington, D.C., CD-ROM.

Kim, K. e L. R. Rilett (2001) Genetic-Algorithm-Based Approach for Calibrating Microscopic Simulation Models. In: Proceedings of the Intelligent Transportation Systems Conference 2001, Oakland,USA, IEEE, p. 698-704.

Koorey, G. (2002) Assessment of rural road simulation modeling tools. In: IPENZ Transportation Group Technical Conference 2002. Disponível em http://ipenz.org.nz/test/ ipenztg_cd/cd/2002pdf/23_koorey_simulation.pdf. Acesso em 28/jan/2004.

Lee, D., X. Yang e P. Chandrasekar (2001) Parameters calibration for PARAMICS using genetic algorithms. In: Transportation Research Board $80^{\text {rd }}$ Annual Meeting, Washington, D.C., CD-ROM.

Ma, T. e B. Abdulhai (2002) Genetic Algorithm-Based Optimization Approach and Generic Tool for Calibrating Traffic Microscopic Simulation Parameters. Transportation Research Record 1800, p. 6-15. 
Ma, T. e B. Abdulhai (2001) Genetic Algorithm-Bàsed Combinatorial Parametric Optimization for the Calibration of Microscopic Traffic Simulation Models. In: Proceedings of the Intelligent Transportation Systems Conference 2001, Oakland, USA, IEEE, p. 846-853.

McLean, J. R. (1989) Two-Lane Highway Traffic Operations Theory and Practice. New York, Gordon and Breach Science Publishers.

Rilett, L. R. e K. Kim (2001) Automatic Calibration of the Surface Transportation and Supply Models Using ITS Data. Proceedings of the $9^{\text {th }}$ World Congress on Transport Research, Seoul, Coréia. (Cópia fornecida pelos autores).
Robertson, H. D.; J. E. Hummer e D. C. Nelson (1994) Manual of Transportation Engineering Studies. New Jersey, PrenticeHall, Inc..

Romana, M. G. (2004) Evaluación práctica de niveles de servicio em carreteras convencionales de dos carriles em España. Disponível em: http://www.caminos.upm.es/ict/ 2cartesi.htm. Acesso em 31/jan/2004.

Rohatgi, V. K. (1976) An Introduction to Probability Theory and Mathematical Statistics. John Wiley \& Sons, USA.

Wall, L.; T. Christiansen e J. Orwant (2000) Programming Perl. California, O'Reilly \& Associates.

\section{CONTATOS}

${ }^{1}$ Nome: Cintia Yumiko Egami

${ }^{2}$ Nome: José Reynaldo Setti

E-mail: cintiaye@sc.usp.br

\footnotetext{
E-mail: jrasetti@usp.br
}

${ }^{3}$ Nome: Laurence R. Rillet

E-mail: 1rillet2@unl.edu 